\title{
STRUCTURE-PROPERTY OF WET-SPUN ALGINATE-BASED PRECURSOR FIBERS MODIFIED WITH NANOCARBONS
}

\author{
Grzegorz Szparaga*, Magdalena Brzezińska, Ewelina Pabjańczyk-Wlazło, \\ Michał Puchalski, Sławomir Sztajnowski, Izabella Krucińska
}

Department of Material and Commodity Sciences and Textiles Metrology, Faculty of Material Technologies and Textile Design, Lodz University of Technology, Zeromskiego Str. 116, 90-924 Lodz, Poland

*Corresponding author email: grzegorz.szparaga@p.lodz.pl

\begin{abstract}
:
The results of studies assessing the influence of the addition of carbon nanofillers, such as multiwalled carbon nanotubes (MWCNTs) and graphene oxide (GO) that differ in size and structure, on the molecular and supramolecular structure and properties of alginate fibers that might be prospective precursors for carbon fiber (PCF) industry are presented in this article. The investigation was carried out by Fourier transform infrared (FTIR) spectroscopy, wide-angle $X$-ray diffraction (WAXD), and tension testing. In the frame of the current study, two types of alginate fibers were examined and compared: alginic acid and calcium alginate fibers. Alginic acid fibers were formed by chemical treatment of calcium alginate fibers with hydrochloric acid due to the fact that $\mathrm{Ca}^{2+}$ ions presented in the fibers were expected to adversely affect the prospective carbonization process. This investigation brought important conclusions about the influence of nanofillers on the physical properties of the final material. Understanding the link between the incorporation of carbon nanostructures and a possible influence on the formation of ordered carbon structures in the precursor fibers brings an important opportunity to get insights into the application of alginate fibers as a prospective base material for obtaining cost-efficient carbon fibers.
\end{abstract}

\section{Keywords:}

precursor fibers, alginic acid, calcium alginate, wet-spinning, carbon nanotubes, graphene, nanomaterials

\section{Introduction}

Man-made fibers composed of natural polymers have attracted increasing attention in materials engineering and novel technologies. They are used in a wide range of solutions in many industries, both as strengthening elements for classical polyolefin materials and as systems with special functional properties [1]. Among the technologically interesting polymers with wide application potential, alginates are noteworthy. Alginates are linear polysaccharides made of $B$-D-mannuronic (M) and $\alpha$-l-guluronic (G) acids and can have widely varying compositions and unit sequence configurations [2,3]. An important aspect affecting the polymeric properties is the interaction between neighboring carboxylic groups in a-Iguluronic acid in the presence of a bivalent metal ion, such as calcium, copper, or magnesium. The zigzag structure of a-Iguluronic acid makes it possible to join two neighboring parallel repeat units with a chemical bond generated by a bivalent metal ion, resulting in a so-called "egg-box" structure [4]. The possibility to create various structural forms of alginate and thus to obtain materials with different physicochemical properties is also very interesting from a scientific standpoint [5].

In the current study, a wet-spun method of obtaining fibers was elected due to the type of the selected polymer, which requires dissolving in a solvent to be spun. In this process, fibers are extruded directly into a liquid bath, called coagulation bath, which is a non-solvent to the polymer solution and then have to be in order to orient the polymers to give the fiber its strength.
Using the special composition of coagulation bath in the wetspinning technique, one can obtain a range of different alginate fibers incorporating copper, sodium, or calcium or fibers made of alginic acid. Currently, alginate fibers are widely applied in medicine, primarily as dressing materials [6-12]. Due to their swelling and gelling properties, such dressings do not adhere to the wound and can easily be removed. Moreover, alginatebased medical products can also be used for controlled drug release $[13,14]$ as well and in many other areas of regenerative medicine [15-22]. Unfortunately, their low strength and high hydrophilicity are major challenges for the use of alginatebased materials in technical applications. However, one characteristic feature makes them interesting in other areas of investigation. Given that their structure is similar to cellulose, alginate fibers can act as precursors for carbon fibers (PCFs). Currently, carbon fiber technology is based on two types of precursors (polyacrylonitrile fibers and mesophase pitches), both of which are quite harmful to the natural environment. Therefore, it seems preferable to use a natural precursor, which is abundantly available and cheap to obtain carbon fibers with specific functional properties.

In analyzing the carbonization process, one should remember that strength properties largely depend on the supramolecular and molecular structure, porosity, and strength of the precursor fibers. Given that the tensile strength of alginate fibers is much lower than that of polyacrylonitrile fibers, the resulting carbon fibers are suitable only for specific technical application such as absorptive materials or applications that do not require 
high strength. Nanotechnology can be important in the manufacture of carbon fibers from alginate precursors. Thus, it is expected that the functionalization of the precursor fibers with nanomaterials could result in changes in the physical and chemical characteristics of the final carbon fibers. The functionalization can be achieved among others through the incorporation of carbon nanoadditives, such as carbon nanotubes, graphene, or fullerenes, which can lead to several integrated carbon conformations, giving rise to oriented crystalline structures obtained with high process efficiency. It is known from the literature that polymers modified with carbon structures can exhibit a number of interesting properties. The use of nanotubes, graphene, and fullerenes enables the production of materials with conductive, biochemical, adsorptive, and other qualities [23-27].

The main objectives of the current study were to determine the effect of the amount of carbon nanoadditives on the molecular and supramolecular structure of prospective alginate PCFs and assess its role in forming the properties of nanocomposite alginate fibers obtained by large-scale wet-spinning processes that might be applied as PCFs. The current study is focused on the formation of alginate fibers containing graphene oxide (GO) and multiwalled carbon nanotubes modified with hydroxyl groups (MWCNT-OH). The selection of the specific nanoadditives was based on the structural differences that might affect the final characteristics of the fibers ("flakes" of $\mathrm{GO}$ versus functionalized carbon nanotubes of more complex three-dimensional [3D] structure). This in turn allows analyzing the influence of the size and shape of the carbon nanofiller on the structure and properties of the PCFs. The results of this investigation were obtained by analytical techniques such as wide-angle X-ray diffraction (WAXD) and Fourier transform infrared (FTIR) spectroscopy, with the aim to track the changes in the molecular and supramolecular structures of alginic acid and calcium alginate fibers during large-scale spinning processes along with the influence of selected carbon nanomaterials on these structures.

The results of this article are a part of the project titled "Alginate fibers modified with carbon nanostructures as a precursor for obtaining fibrous carbon materials" which was financed by the National Science Centre in Poland. Understanding the link between the incorporation of carbon nanostructures and a possible influence on the formation of ordered carbon structures in PCFs brings an important opportunity to advance the understanding of functionalization of alginic fibers which are a prospective base material for obtaining cost-efficient carbon fibers. It is the first part of the study that addresses several important areas which serves as a prelude for undertaking a longitudinal analysis of alginate-based resources in applications for carbon fiber industries.

\section{Experimental}

\subsection{Materials}

Protanal LF 10/60 LS sodium alginate (FMC Biopolymer, Norway) was used in the study as a basic polymeric material.
The following carbon nanoadditives were used:

2.1.1. MWCNTs with a diameter of $10-20 \mathrm{~nm}$ and a length of $0.5-2 \mathrm{~mm}, 95 \%$ purity, and surface area of $200 \mathrm{~m}^{2} / \mathrm{g}$ (Nanostructured \& Amorphous Materials Inc., USA).

2.1.2. $\mathrm{GO}$ as a single-layer $\mathrm{GO}$ dispersion of $5 \mathrm{~g} / 1,000 \mathrm{~mL}$ in aqueous suspension; composition: carbon $79 \%$; oxygen $20 \%$; flake size $0.3-0.7 \mathrm{~mm}$; thickness of one atomic layer minimum $80 \%$ (Graphene Supermarket, USA).

\subsection{Methods}

\subsubsection{Rheological properties of spinning solutions}

The rheological properties of alginate spinning solutions in distilled water were determined using a RheolabQC (Anton Paar $\mathrm{GmbH}$, Austria) rotary rheometer at $20^{\circ} \mathrm{C}$ with an $\mathrm{H}$-type cylinder. This type of the cylinder is specifically required for measuring rheological properties of non-Newtonian fluids that are dependent on the relevant shear stress and timescales as well as viscosity of the sample. The rheological parameters " $n$ " and " $k$ " were estimated on the basis of the flow curves according to the Ostwald de Waele equation:

$$
\tau=k \cdot \gamma^{n}
$$

where $t$ is the shear stress and $g$ is the shear rate.

\subsubsection{Molecular structure measurements}

The molecular structures of the alginate fibers were analyzed using an FTIR spectrometer, Nicolet 6700 (Thermo Scientific, USA), in attenuated total reflectance (ATR) mode with a diamond crystal (incidence angle $45^{\circ}$ ). The measurement conditions were as follows: resolution was $2 \mathrm{~cm}^{-1}$, range of infrared (IR) radiation was 4,000-600 $\mathrm{cm}^{-1}$, and 32 scanning procedures of each sample by IR radiation were carried out to obtain absorption spectra. The absorption spectra of the fiber preparations under investigation were plotted in the system: $A=f(1 / l)$ as a basis for the interpretation of the molecular fiber structure.

\subsubsection{Supramolecular structure measurements}

Supramolecular structures of the alginate fibers were studied by a wide-angle X-ray diffractometer (WAXD), X'Pert PRO (PANalytical, the Netherlands), using a Cu Ka source $(I=0.154 \mathrm{~nm})$ and the following tube parameters: $40 \mathrm{kV}$ accelerating voltage and $30 \mathrm{~mA}$ anode current. An X'Celerator semiconductor detector was used for the detection of the X-ray spectrum. Diffraction profiles of powdered fiber samples were taken over the $2 \theta$ range of $5-60^{\circ}$. The obtained X-ray diffractograms were numerically analyzed using WAXSFIT software $[28,29]$

\subsubsection{Mechanical measurements}

The mechanical properties of the obtained fibers, such as the tenacity and elongation at break, were determined using 
an Instron 5944 mechanical testing machine (Instron, USA). Measurement was carried out based on the PN EN ISO 2062:2010 standard. The distance between the clamps was $100 \mathrm{~mm}$, and the displacement rate was $100 \mathrm{~mm} / \mathrm{min}$. The linear mass of the multifilament was measured using a WTB 200 balance (Radwag, Poland) and the PN ISO 1973:1997 standard.

\subsubsection{Nanoadditive dispersion}

The analysis of the distribution of nanoadditives in the alginate fiber material was based on images obtained using the TECNAI SuperTWIN FEG $(200 \mathrm{kV}$ ) transmission microscope (FEl, USA). The samples in the form of thin films for observation in the transmission microscope were prepared using LEICA microtome (cut sections with 50-nm increments). Observations were made using diffraction contrast in the bright-field mode, the so-called bright field and phase contrast, and the so-called high-resolution electron microscopy (HREM).

\subsubsection{Determination of the share of calcium ions in fibers}

The calcium content in the tested samples of alginate fibers after treatment with hydrochloric acid was determined by the atomic emission spectroscopy method using the flame technique. The Thermo Scientific iCE 3500 AAS spectroscope was used for the study. The delivered sample was subjected to microwave mineralization based on PN-EN-16711-1. The mineralization process was carried out in the system of nitric acid-hydrogen peroxide using Milestone Start D microwave mineralizer.

\subsection{Processes}

\subsubsection{Fiber-spinning process}

Calcium alginate fibers were obtained by wet-spinning method from an aqueous solution of sodium alginate. $7.4 \%$ aqueous solutions of sodium alginate were used for spinning (this concentration was selected based on the previous research) [30]. The solutions were made by dissolving the polymer in water and stirring mechanically for approximately $4-5 \mathrm{~h}$. Fibers were formed using a 500-hole spinneret with a 0.08 $\mathrm{mm}$ hole diameter. Fibers were coagulated in a $3 \% \mathrm{~m} / \mathrm{m}$ aqueous solution of calcium chloride at $15^{\circ} \mathrm{C}$. The drawing process was conducted in the same type of solution but at $60^{\circ} \mathrm{C}$. Subsequently, the fibers were washed in distilled water and freeze-dried. The optimum fiber-forming conditions were determined based on preliminary studies [31-33]. They were selected to obtain fibers with the best strength properties and to prevent the breaking of elementary fibers in the bundle, thus ensuring process stability.

The alginic acid fibers were formed by chemical treatment of the calcium alginate fibers with a $2 \%$ aqueous solution of hydrochloric acid for $24 \mathrm{~h}$. Subsequently, the fibers were washed in distilled water and freeze-dried. In this case, a modifying bath module of 1:200 in relation to the weight of the fiber was used. The fiber was then rinsed repeatedly in distilled water until the chloride ions from the acid were removed. As a result of this modification, the share of calcium in alginate determined by emission atomic spectrometry dropped from $5 \%$ to $0.05 \%$.

\subsubsection{Dispersion of the nanoadditives in the polymer matrix}

For fibers containing MWCNTs, polymer dissolution was preceded by the suspension of nanoadditives in water, which was achieved using a Bandelin Sonopuls HD 2200 ultrasonic probe (Germany) for $30 \mathrm{~min}$. The polymer was added to the suspension, and the system was mixed using a mechanical stirrer for approximately 4-5 h. For fibers with GO, the polymer was dissolved in a diluted suspension of $G O$ and mixed with a mechanical stirrer for approximately $4-5 \mathrm{~h}$.

\section{Results and discussion}

\subsection{Rheological properties of spinning solutions}

Achieving the appropriate apparent dynamic viscosity of the spinning solution, by adjustment of different solution characteristics (polymer content, solvent systems, etc.), is very important in the process of fiber formation by wet spinning from solution. This is due to the fact that this property should ensure the process stability. In our previous works [31-33], we determined the concentrations of the spinning solution that resulted in apparent dynamic viscosities in the range of 30 60 Paxs which was confirmed to provide the stable spinning process. At the same time, it was found that the addition of non-fiber-forming nanoadditives changed the rheological parameter $k$ in the Ostwald de Waele equation, affecting the consistency of the solution. Table 1 summarizes the effects of the introduction of carbon nanoadditives on the rheological characteristics. The addition of $2 \%$ by the weight of the polymer of different nanofillers to the spinning solution led to a decrease in the rheological parameters $n$ and $k$. The lowest $n$ was found in the solution containing GOs, probably due to strong interactions between the surface of the nanoadditives and the

Table 1. Rheological parameters " $n$ " and " $k$ " for $7.4 \% \mathrm{~m} / \mathrm{m}$ alginate sodium solutions

\begin{tabular}{|c|c|c|c|}
\hline \multirow{2}{*}{ Sample symbol } & Solution & $\mathbf{2}$ & Rheological parameters \\
\cline { 3 - 4 } & & $\mathbf{n}(-)$ & $\mathbf{k}$ (Paxs) \\
\hline Alginate & Sodium alginate & 0.72 & 36.68 \\
\hline Alginate + MWCNT & Sodium alginate + 2\% MWCNT & 0.71 & 34.09 \\
\hline Alginate + GO & Sodium alginate + 2\% GO & 0.68 & 32.23 \\
\hline
\end{tabular}

Abbreviations: GO, graphene oxide; MWCNT, multiwalled carbon nanotube 
macromolecules of the polymers, making the solutions more strongly polymeric.

Figure 1a shows the changes in the apparent dynamic viscosity as a function of shear rate, while Figure $1 \mathrm{~b}$ shows the dependence of shear stress as a function of shear rate.

The analysis of flow curves (Figure 1b) indicates that all solutions are shear-thinning non-Newtonian liquids $(n<1)$ without a flow limit. The shear stress increases nonlinearly with the shear rate, and the flow curves pass through the origin of the coordinate system. Changes in the parameter $n$ are also correlated with the rheological parameter $k$, which is the highest for the solution without nanoadditives.

\subsection{FTIR results of the molecular structure study}

Differences in the molecular structures of both types of alginate fibers (calcium and sodium alginates) with and without additives are shown in the spectrograms (Figure 2). In Figure $2 a$, the FTIR spectrum of the calcium alginate fibers shows the following characteristic bands: a peak at $3,252 \mathrm{~cm}^{-1}$ attributable to stretching vibrations of the $\mathrm{O}-\mathrm{H}$ group, two signals corresponding to stretching vibrations of the $\mathrm{C}-\mathrm{H}$ and $\mathrm{C}-\mathrm{H}_{2}$ groups at 2,923 $\mathrm{cm}^{-1}$ and $2,854 \mathrm{~cm}^{-1}$, and bands attributable to asymmetrical (at $1,589 \mathrm{~cm}^{-1}$ ) and symmetrical (at $1,412 \mathrm{~cm}^{-}$ $\left.{ }^{1}\right)$ stretching vibrations of the $\mathrm{COO}^{-}$group. Furthermore, the spectrum of the calcium alginate fibers exhibits bands attributable to stretching vibrations of the $\mathrm{C}-\mathrm{O}$ group $\left(1,300 \mathrm{~cm}^{-1}\right)$; superimposed signals of the stretching vibrations of the $\mathrm{C}-\mathrm{O}, \mathrm{C}-\mathrm{C}$, and $\mathrm{C}-\mathrm{C}-\mathrm{C}$ groups $\left(1,250-1,000 \mathrm{~cm}^{-1}\right)$; and bending vibrations assigned to the $\mathrm{O}-\mathrm{H}$ bond $\left(1,300 \mathrm{~cm}^{-}\right.$ $\left.{ }^{1}\right)$. In turn, the region of $850-600 \mathrm{~cm}^{-1}$ also displays peaks attributable to twisting vibrations of the $\mathrm{C}-\mathrm{O}$ group. Following the hydrochloric acid modification, the spectrum of the fibers shifted toward longer wavelengths (Figure $2 b$ ). For instance, the maximum stretching vibrations of the $\mathrm{O}-\mathrm{H}$ group moved to $3,410 \mathrm{~cm}^{-1}$. Moreover, the peaks corresponding to symmetrical and asymmetrical stretching vibrations of the $\mathrm{COO}^{-}$group became markedly weaker, and intense peaks corresponding to stretching vibrations of the $\mathrm{C}-\mathrm{H}$ bond appeared. Notably, more intense peaks are observed below $1,000 \mathrm{~cm}^{-1}$.

Modification of the calcium alginate fibers by nanocarbon materials resulted in an insignificant decrease in the intensity of the band attributable to stretching vibrations of the $\mathrm{O}-\mathrm{H}$ bond located at $3,252 \mathrm{~cm}^{-1}$ along with a significant increase in the intensity of the two signals corresponding to stretching
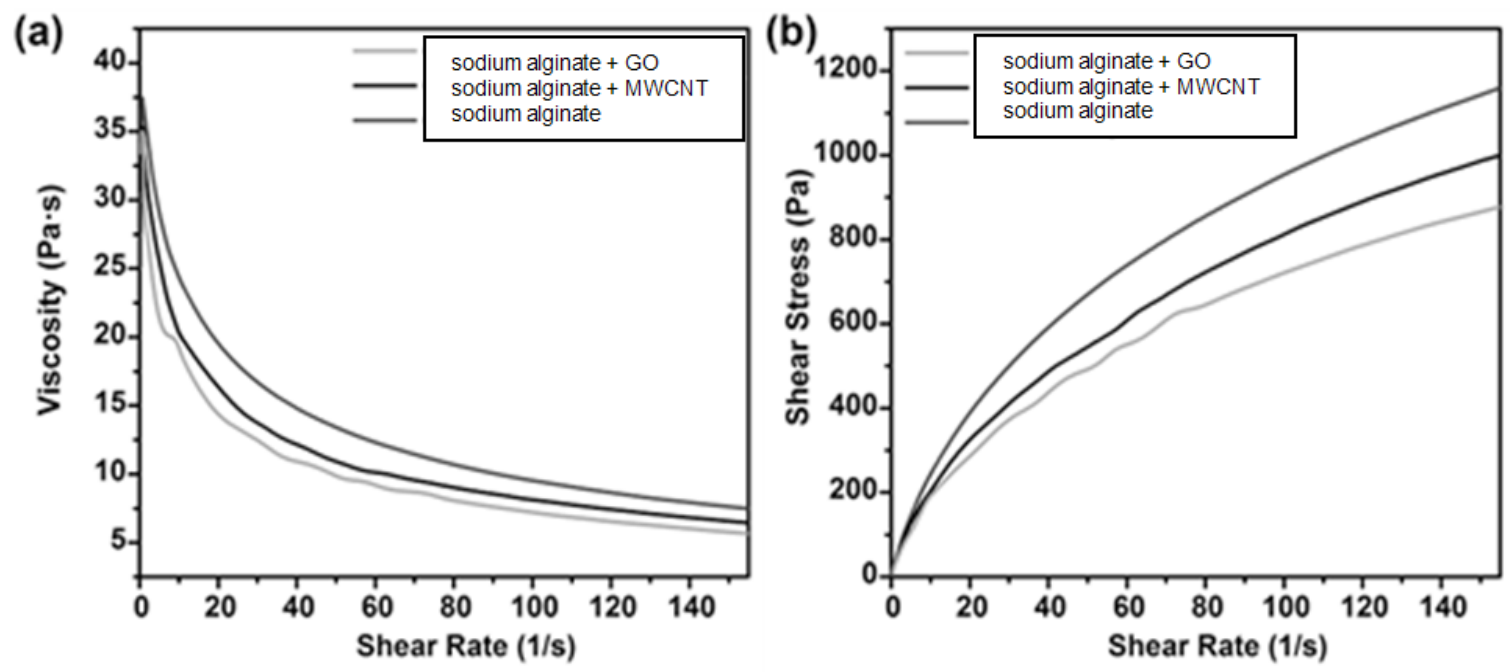

Figure 1. Apparent dynamic viscosity as a function of shear rate (a) and flow curves (b) of sodium alginate solutions

(a)

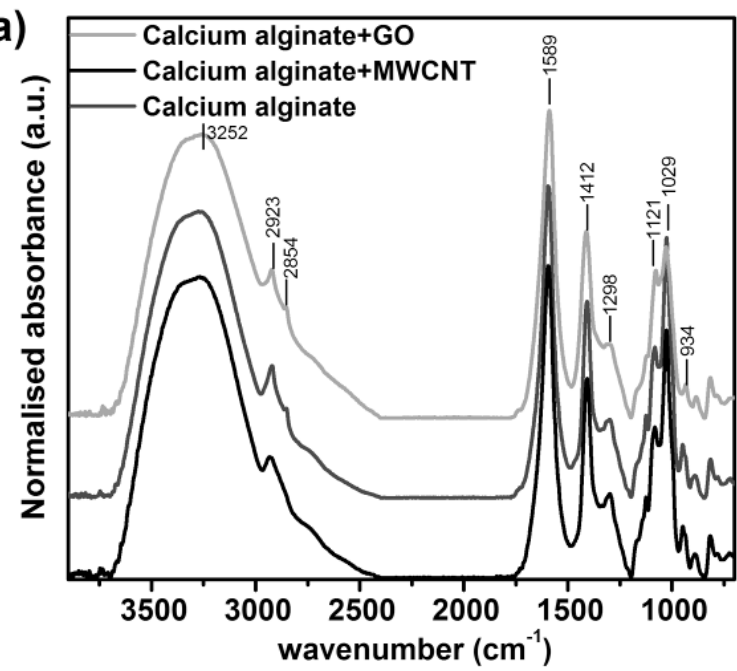

(b)

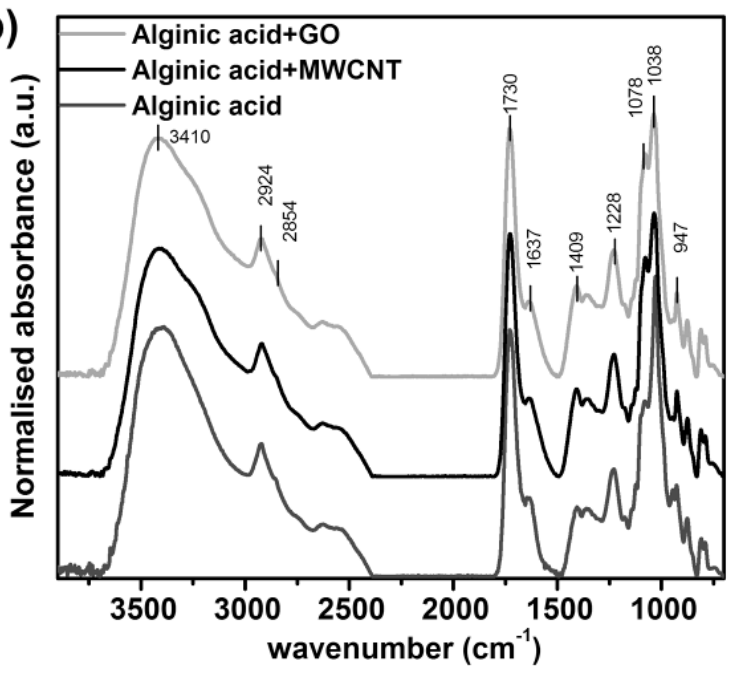

Figure 2. Spectrograms of the studied alginate fibers: (a) calcium alginate fibers; (b) alginic acid fibers 
vibrations of the $\mathrm{C}-\mathrm{H}$ and $\mathrm{C}-\mathrm{H}_{2}$ groups at $2,923 \mathrm{~cm}^{-1}$ and $2,854 \mathrm{~cm}^{-1}$. Furthermore, after $\mathrm{GO}$ addition, changes in the intensity of the superimposed signals of stretching vibrations of the C-O, C-C, and C-C-C groups $\left(1,250-1,000 \mathrm{~cm}^{-1}\right)$ were more visible than in the case of MWCNT addition. The observed changes in the FTIR spectra verified the presence of chemically functionalized carbon materials inside the polymer matrix without higher-order molecular changes in the calcium alginate structure. The analysis of the influence of carbon nanomaterial addition on the molecular structure of alginic acid led to similar conclusions. An insignificant decrease in the intensity of the band attributable to stretching vibrations of the $\mathrm{O}-\mathrm{H}$ bond located at $3,252 \mathrm{~cm}^{-1}$ and a significant increase in the intensity of the two signals corresponding to stretching vibrations of the $\mathrm{C}-\mathrm{H}$ and $\mathrm{C}-\mathrm{H}_{2}$ groups at $2,923 \mathrm{~cm}^{-1}$ and $2,854 \mathrm{~cm}^{-1}$ were observed. Furthermore, after $\mathrm{GO}$ addition, changes in the intensity of the superimposed signals of stretching vibrations of the $\mathrm{C}-\mathrm{O}, \mathrm{C}-\mathrm{C}$, and $\mathrm{C}-\mathrm{C}-\mathrm{C}$ groups $\left(1,250-1,000 \mathrm{~cm}^{-1}\right)$ were more visible than in the case of MWCNT addition.

\subsection{WAXD results of the supramolecular structure study}

The influence of carbon nanoadditives on the supramolecular structure of alginate was analyzed by the wide-angle X-ray scattering method. The diffraction profiles of the alginic acid fibers (Figure $3 a$ ) and calcium alginate fibers (Figure $3 b$ ) are shown in Figure 3.

According to the recent works of Arnott et al. [33] and Fabia et al. [34], the supramolecular structure of alginate depends on its chemical composition and on the positions and lengths of the polymannuronic (M) and polyglucuronic acid $(\mathrm{G})$ blocks. As shown in Figure $3 a$, the diffraction profiles of the alginic acid

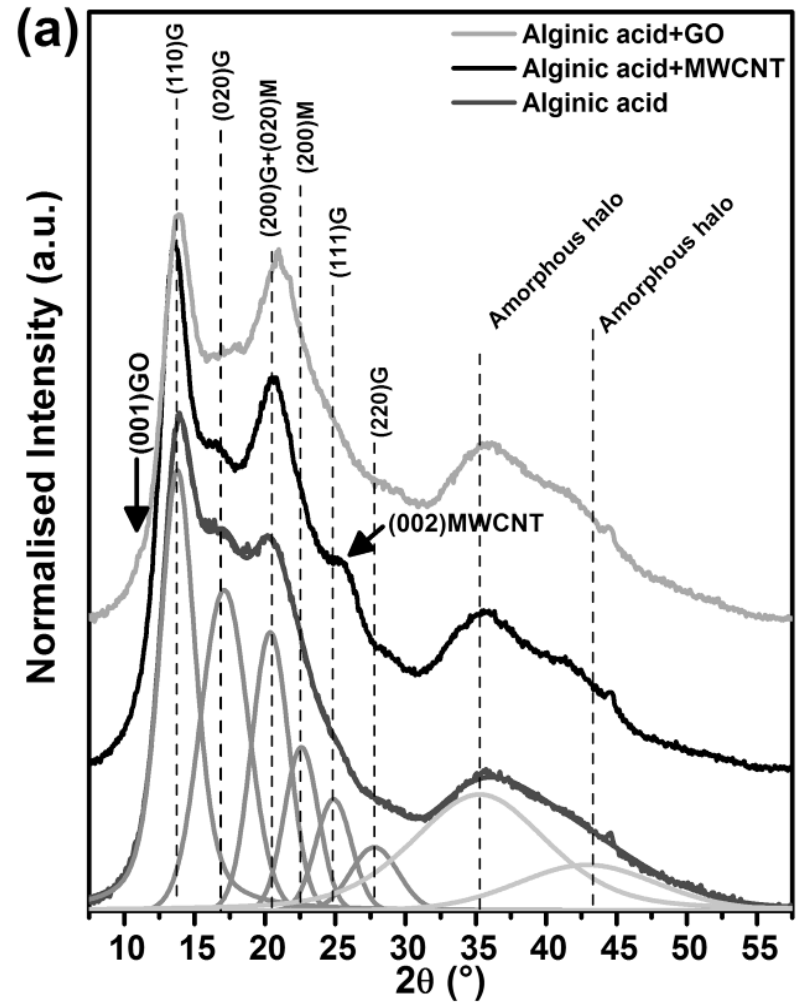

fibers are the compilation of the signals for polymannuronic acid, which are indexed as a two-chain orthorhombic unit cell with dimensions of $a=0.76 \mathrm{~nm}, b=0.86 \mathrm{~nm}$, and $c=1.04 \mathrm{~nm}$ (chain direction) and the signals for polyglucuronic acid, which are indexed as a two-chain orthorhombic unit cell with dimensions of $a=0.86 \mathrm{~nm}, b=1.07 \mathrm{~nm}$, and $c=0.87 \mathrm{~nm}$ (chain axis) in space group $\mathrm{P} 2,2,2,{ }_{1}{ }^{34}$ As shown in Figure $3 a$, diffraction profiles contain clear crystalline peaks in the range of $2 \theta$ $5-30^{\circ}$ corresponding to the (110), (020), (200), (111), and (220) crystalline planes of guluronan and (020) and (200) crystalline planes of mannumoronan. The addition of surface-modified MWCNTs and GOs to the fibers resulted in the appearance of an additional peak in the diffraction profile located at $2 \theta$ values of $25.8^{\circ}$ and $10.0^{\circ}$, respectively. These peaks corresponded to the (001) planes of graphite walls of carbon nanotubes and graphite layers of GOs.

The diffraction profiles of the calcium alginate fibers (Figure 3b) have different shapes than those of the alginic acid fibers (Figure 3a). The presence of $\mathrm{Ca}^{2+}$ ions in the alginate matrix leads to the creation of an egg-box structure of the polymannuronic and polyglucuronic acid blocks. The diffraction profiles contain two amorphous halos with maxima located at $2 \theta$ values of $22.0^{\circ}$ and $42.5^{\circ}$ and a peak corresponding to the diffraction of the crystalline structure with a maximum located at approximately $13^{\circ}$. According to the previous works, the visible diffraction maximum is a superposition of the diffraction peaks of the specific crystalline structure, i.e., the egg-box structure, of polymannuronic acid and polyglucuronic acid [32]. As in the case of alginic acid fibers, the WAXD profile of the calcium alginate fibers contains characteristic peaks in the range of $10-20^{\circ}$ corresponding to the (010), (001), (110), (011), (101), and (100) crystalline planes of guluronan and the

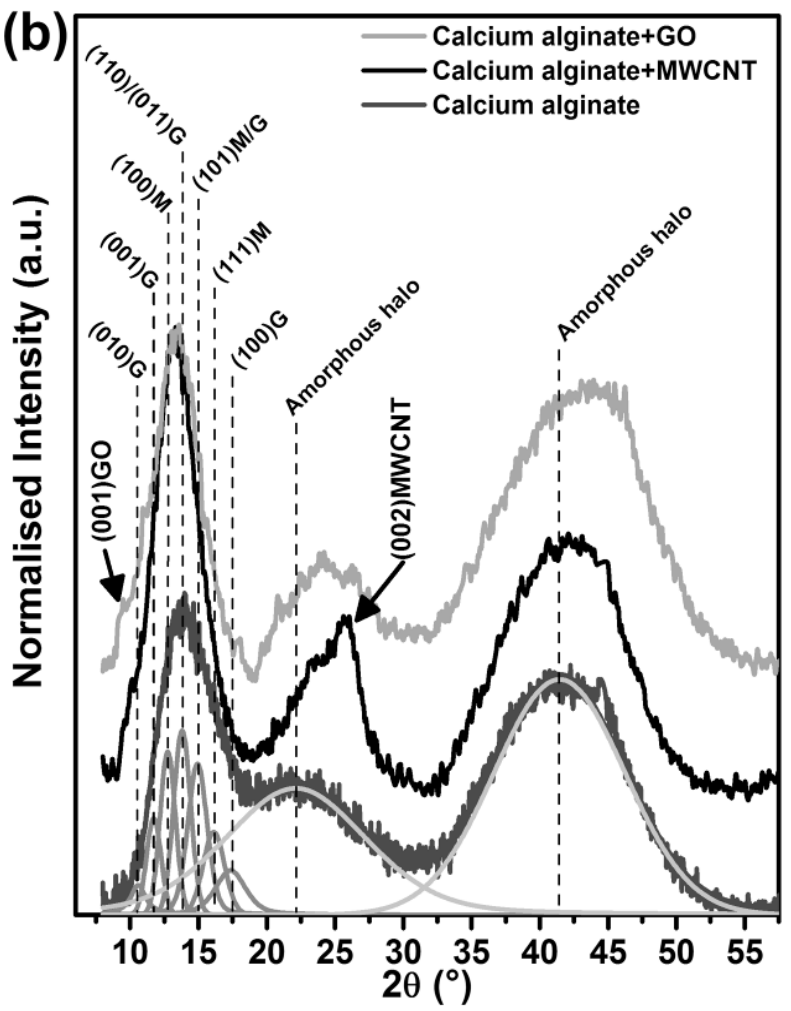

Figure 3. Diffraction profiles of fibers containing nanoadditives: alginic acid fibers (a); calcium alginate fibers (b) 
(100), (101), and (111) crystalline planes of mannumoronan. The intensity, position, and full width at half-maximum of the crystalline peaks suggest that the egg-box structure is a highly dispersed structure with ordered regions.

More detailed analysis of the differences in the supramolecular structures of the various alginic acid and calcium alginate fiber samples was conducted using WAXSFIT software. Numerical analysis provided a quantitative estimation of the influence of carbon nanoadditives on the supramolecular structure. According to the Hindeleh and Johnson method, the obtained diffraction profiles were deconvoluted into amorphous halos and crystalline peaks approximated by numerically calculated Gauss and Lorentz functions. The crystalline phase content in the studied materials was calculated according to the following equation:

$$
\chi_{\mathrm{C}}(\%)=\frac{A_{\mathrm{C}}}{A_{\mathrm{C}}+A_{\mathrm{A}}} \times 100
$$

where $A_{\mathrm{A}}$ and $A_{\mathrm{C}}$ are the integral intensities of the amorphous halo and the peaks originating from the crystalline phase, respectively.

The size of the crystalline area was determined by measuring the width of the diffraction peak and using Scherrer's formula:

$$
L_{(h k l)}=\frac{K \lambda}{B \cos \theta}
$$

where $L_{(\mathrm{hkl})}$ is the average size of the crystalline area perpendicular to the lattice plane $(h k l), q$ is the Bragg angle for the plane $(h k l), I$ is the $X$-ray radiation wavelength (for $\mathrm{Cu} \mathrm{Ka}$, $I=0.15418 \mathrm{~nm}), B$ is the half-width of the diffraction peak for the plane $(h k l)$, and $K$ is Scherrer's constant, which for polymers is equal to 0.9 . The last characteristic feature of the structure formed with different carbon nanoadditives was detected by the analysis of the lattice length ( $d$-spacing) calculated according to Bragg's equation:

$$
d_{(h k l)}=\frac{\lambda}{2 \sin \theta}
$$

where $I$ is the wavelength of the X-ray source $(0.15418 \mathrm{~nm})$ and $q$ is the angle of reflection (half of $2 \theta$ of the peak position).

The size of the crystalline area and the lattice length were calculated for the most intense diffraction peaks corresponding to the (110) crystalline plane of guluronan and the (200) crystalline plane of mannumoronan in alginic acid and the (110)/ (011) crystalline plane of guluronan and the (100) crystalline plane of mannumoronan in calcium alginate. The values of the calculated factors are summarized in Table 2.

Based on the results summarized in Table 1, the magnitude of the effect of carbon nanomaterial addition on the supramolecular structure of the studied alginate fibers was determined.

For the alginic acid fibers, the addition of MWCNTs and GO resulted in an insignificant increase in the degree of crystallinity but significant changes in the values of the factors that defined the crystallites. The distance between the lattice planes, calculated by the $d$-spacing, increased for both the guluronan and mannumoronan structures. The analysis of the influence of the addition of carbon additives on the average size of the crystalline area in the alginic acid structure clearly shows an increase in $L_{(\mathrm{hkl})}$ corresponding to the (110) crystalline plane of guluronan and a decrease in $L_{(\mathrm{hkl})}$ corresponding to the (200) crystalline plane of mannumoronan. The analysis of the obtained results suggests the strong impact of additives on the crystalline structure of alginic acid without significantly affecting the overall crystallinity of the material. The presence of crystalline additives allows creating a structure with lower orientation, as confirmed by the increased $d$-spacing, which in turn allows for the reformation of the shape of the crystalline area, which can be observed by the changes in the $L_{(\mathrm{hkl})}$ factor. This observation is interesting and important and enables interpreting the change in the macroscopic physical properties of the fibers, e.g., the mechanical properties that are discussed in the next sections.

The addition of carbon nanomaterials also influences the

\begin{tabular}{|c|c|c|c|c|}
\hline Sample & $(h k l)$ & $L\left({ }_{n k l}\right)(\mathrm{nm})$ & $d_{(h k)}(\mathrm{nm})$ & $X \mathrm{c}(\%)$ \\
\hline \multirow{2}{*}{ Alginic acid } & $(110) \mathrm{G}$ & 2.955 & 0.643 & \multirow{2}{*}{70.2} \\
\hline & (200) M & 3.159 & 0.393 & \\
\hline \multirow{2}{*}{ Alginic acid + MWCNT } & $(110) G$ & 3.433 & 0.656 & \multirow{2}{*}{71.7} \\
\hline & (200) M & 2.812 & 0.401 & \\
\hline \multirow{2}{*}{ Alginic acid + GO } & $(110) \mathrm{G}$ & 3.542 & 0.652 & \multirow{2}{*}{71.6} \\
\hline & $(200) \mathrm{M}$ & 2.534 & 0.401 & \\
\hline \multirow{2}{*}{ Calcium alginate } & $(110) /(011) \mathrm{G}$ & 6.680 & 0.694 & \multirow{2}{*}{22.4} \\
\hline & $(100) \mathrm{M}$ & 6.092 & 0.641 & \\
\hline \multirow{2}{*}{ Calcium alginate + MWCNT } & $(110) /(011) \mathrm{G}$ & 5.765 & 0.737 & \multirow{2}{*}{37.8} \\
\hline & $(100) \mathrm{M}$ & 5.590 & 0.672 & \\
\hline \multirow{2}{*}{ Calcium alginate $+\mathrm{GO}$} & $(110) /(011) \mathrm{G}$ & 6.528 & 0.735 & \multirow{2}{*}{29.2} \\
\hline & $(100) \mathrm{M}$ & 6.394 & 0.672 & \\
\hline
\end{tabular}
supramolecular structure of calcium alginate. An increase in the

Table 2. WAXD results of the calculation of supramolecular structure factors

Abbreviations: GO, graphene oxide; MWCNT, multiwalled carbon nanotube; WAXD, wide-angle X-ray diffraction 
degree of crystallinity is clearly visible and is most significant with the addition of chemically functionalized MWCNTs. Similar to alginic acid, the addition of both carbon nanomaterials increases the distance between lattice planes $\left(d_{(h k)}\right)$ in a similar range. The influence of the different carbon materials on the supramolecular structure can be seen in the average size of the crystalline area. The presence of chemically functionalized MWCNTs causes the creation of smaller crystalline areas compared with the addition of GOs - the addition of GO causes a decrease in $L_{(\mathrm{hkl})}$ corresponding to the $(110) /(011)$ crystalline plane of guluronan and an increase in $L_{(\mathrm{hkl})}$ corresponding to the (100) crystalline plane of mannumoronan.

The obtained results suggest that the type and shape of the carbon nanoadditives influence the supramolecular structure of calcium alginate, which is the main difference from alginate acid, where the shape of additives is insignificant. Certainly, the crystalline structure of calcium alginate, i.e., low crystallinity and egg-box structure of the polymannuronic and polyglucuronic acid blocks, is important. Based on these studies, it is difficult to describe exactly how the structure of calcium alginate is ordered, but it is clear that the ordering and crystallization depend on the shape of the additive material, e.g., whether the additive has a lamellar or tubular shape.

\subsection{Nanoadditive dispersion analysis}

Transmission electron microscopy (TEM) and HREM analyses are used to visually evaluate the degree of aggregation of nanofillers in the polymer matrix of the prepared fibers, because the mechanical characteristics of the nanodoped alginic fibers are strongly depended on the degree of dispersion of nanoadditives in the polymer matrix. Nanofiller dispersion is a well-known challenge since carbon nanoadditives express a tendency to agglomerate; however, a good dispersion is an important factor for mechanical reinforcement. The main reason behind agglomeration is the van der Waals attraction and the large surface areas and high surface energy of the nanofillers [35]. For the analysis purposes, the alginic acid fibers were selected, which were obtained directly by the treatment of calcium alginate fibers with $2 \%$ aqueous solution of hydrochloric acid for $24 \mathrm{~h}$. The presence of calcium ions in the fiber structure during the oxidation process and subsequent carbonation might result in disturbances in both processes and structural defects. (a1)

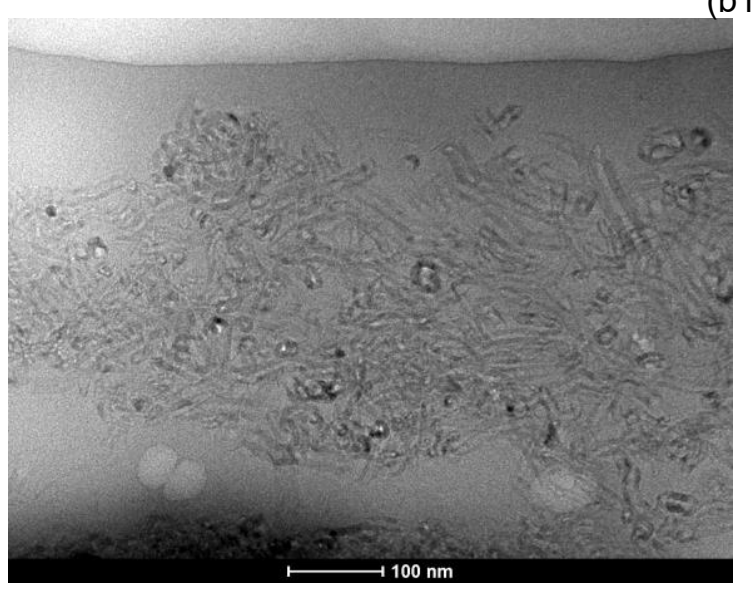

(a2)

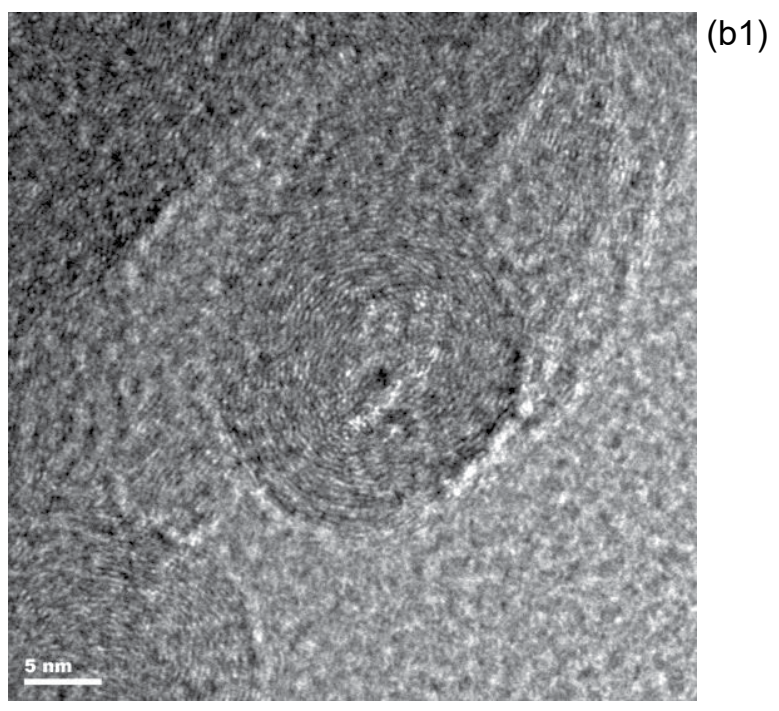

(b1)
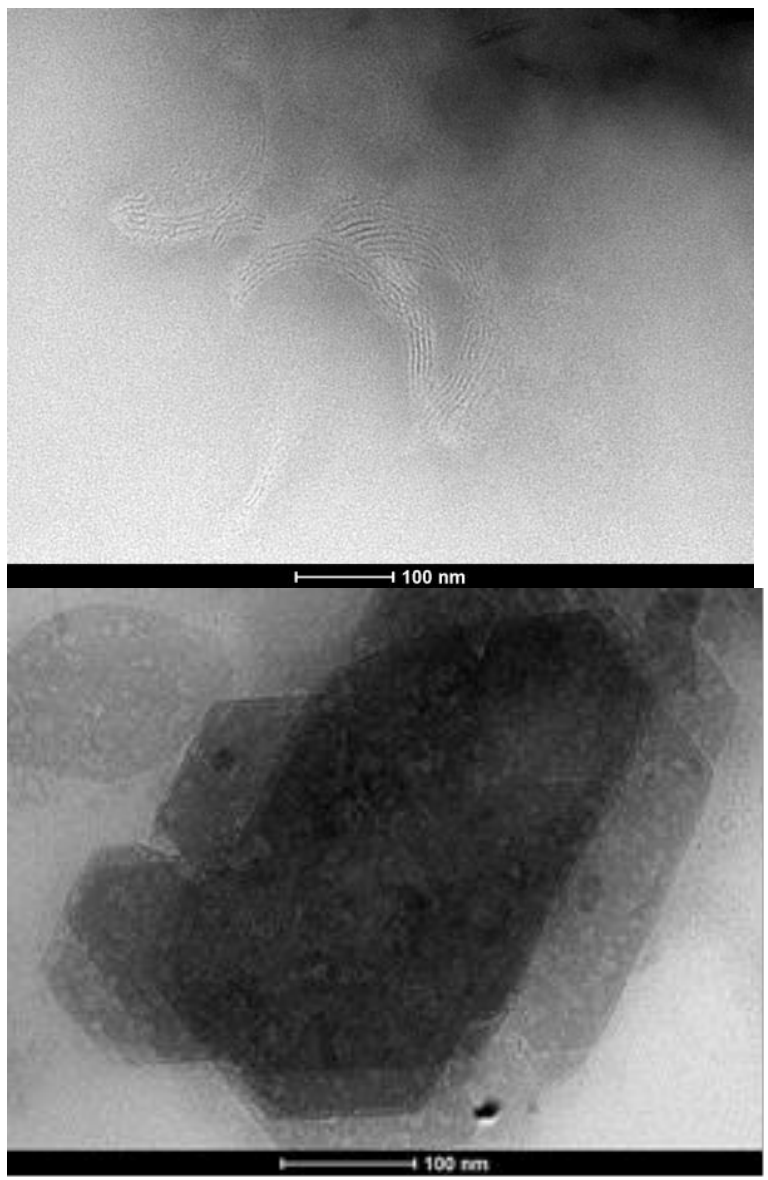

Figure 4. Microscopicanalysis of the distribution of nanoadditives in the fibermaterial(alginicacid): (a1)TEMimage ofMWCNTs dispersed in the polymer matrix;(a2)HREMimageofthecross-section ofMMWCNTs;(b1)TEMimageofthecross-section of GOflakes;(b2)TEMimage oftheaggregatedGOflakes Abbreviations: GO, graphene oxide; HREM, high-resolution electron microscopy; MWCNT, multiwalled carbon nanotube; TEM, transmission electron microscopy 

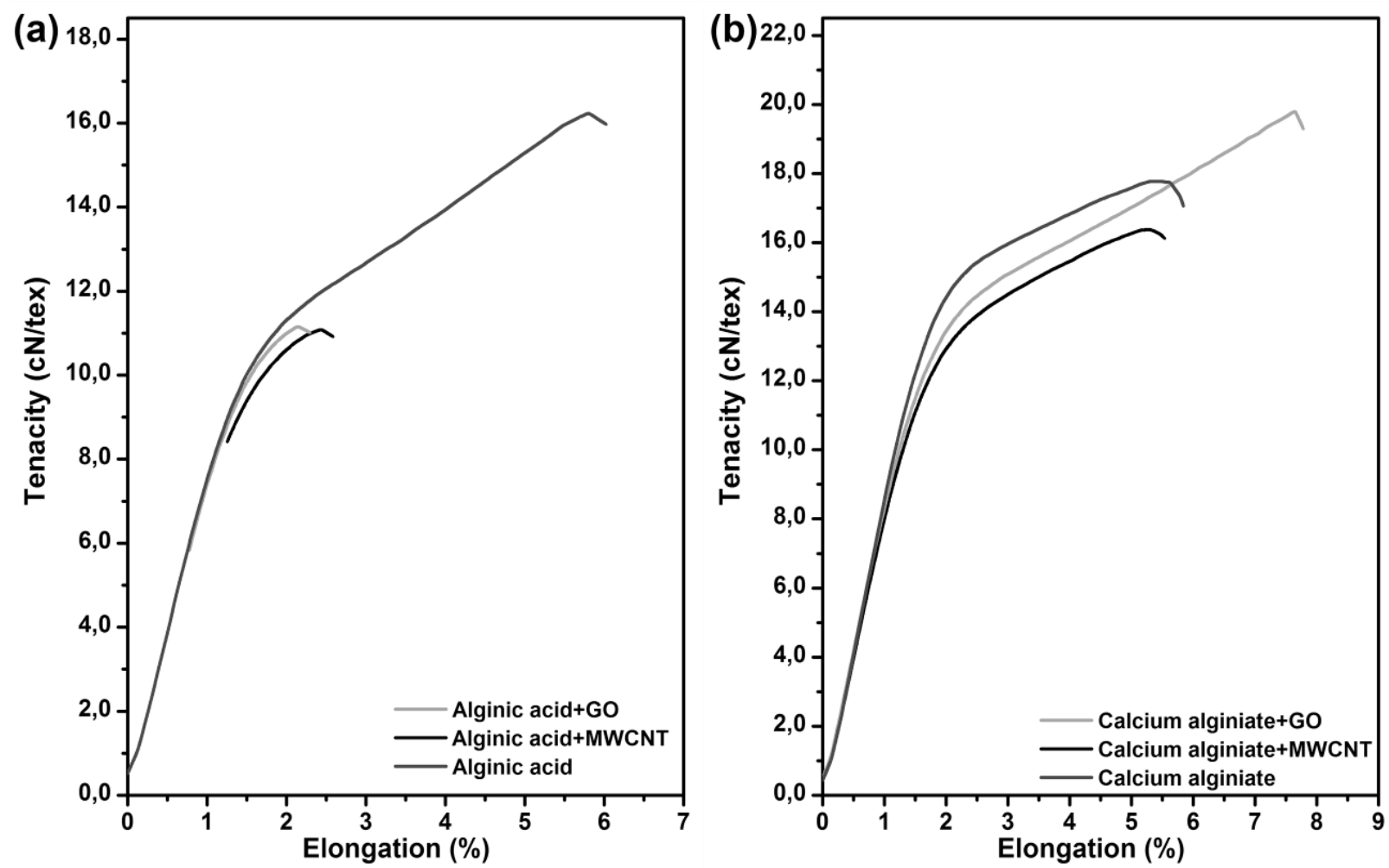

Figure 5. Mechanical profiles of tenacity as a function of elongation for fibers containing nanoadditives: alginic acid fibers (a); calcium alginate fibers (b)

The removal process was assumed not to affect the amount and distribution of nanofillers in the polymer matrix.

The analysis showed that the MWCNTs are averagely homogeneously dispersed on the nanoscale and mostly clusters or agglomerated particles were detected. The analysis also confirmed that MWCNTs are not uniformly orientated parallel to the edge of the fiber. Figure 4(a2) shows the crosssection of MWCNTs that confirm the layered nature of the nanofiller. Figure 4(b1) shows the cross-section of randomly dispersed $\mathrm{GO}$ flakes, aggregated in the layered form. Figure 4(b2) shows the aggregated set of GO flakes. The specific hexagonal structure of the GO flakes was confirmed.

Finally, the analysis showed that both nanofillers were randomly to averagely homogeneously dispersed in the polymer matrix. It indicated that the employed mechanical stirring and the ultrasonic treatment approach were proven to be sufficiently effective approaches to obtain nanodoped composite fibers.

\subsection{Results of the study of physical properties}

The results of the FTIR and WAXD measurements showed the clear influence of the carbon nanomaterials on the molecular and supramolecular structures. The main changes resulting from the addition of carbon materials, i.e., the ordering and crystallization of the alginate material, could strongly impact the physical properties of the fibers, including the mechanical properties; therefore, mechanical factors such as modulus of elasticity, tenacity at break, and elongation at break of the studied materials were analyzed.

Average profiles of the mechanical test measuring the tenacity as a function of elongation are shown in Figure 5. The profiles were calculated by the analysis of $2 \theta$ measurements, and the mean values and standard deviations of the mechanical factors of the studied fibers are summarized in Table 3. For alginic acid, a decrease in the positive mechanical parameters was clearly seen. The unmodified fibers were characterized by the modulus

Table 3. Mechanical properties of calcium alginate fibers and alginic acid fibers formed at an as-spun draw ratio of $80 \%$

\begin{tabular}{|l|c|c|c|c|}
\hline Sample & Linear mass (tex) & $\begin{array}{c}\text { Modulus of } \\
\text { elasticity (cN/tex) }\end{array}$ & $\begin{array}{c}\text { Tenacity at brake } \\
\text { (cN/tex) }\end{array}$ & $\begin{array}{c}\text { Elongation at break } \\
\text { (\%) }\end{array}$ \\
\hline Alginic acid & $54.1 \pm 1.3$ & $7.65 \pm 0.14$ & $16.22 \pm 0.99$ & $5.98 \pm 0.34$ \\
\hline Alginic acid + MWCNT & $57.1 \pm 1.1$ & $7.87 \pm 0.12$ & $11.05 \pm 0.65$ & $2.68 \pm 0.23$ \\
\hline Alginic acid + GO & $60.0 \pm 1.2$ & $7.50 \pm 0.13$ & $10.64 \pm 0.54$ & $2.17 \pm 0.34$ \\
\hline Calcium alginate & $71.2 \pm 2.0$ & $9.01 \pm 0.12$ & $17.96 \pm 0.78$ & $6.63 \pm 0.25$ \\
\hline $\begin{array}{l}\text { Calcium alginate + } \\
\text { MWCNT }\end{array}$ & $75.0 \pm 2.1$ & $8.22 \pm 0.13$ & $16.54 \pm 1.12$ & $5.71 \pm 0.05$ \\
\hline Calcium alginate + GO & $80.1 \pm 2.4$ & $8.50 \pm 0.14$ & $20.42 \pm 1.23$ & $8.71 \pm 0.78$ \\
\hline
\end{tabular}

Abbreviations: GO, graphene oxide; MWCNT, multiwalled carbon nanotube 
of elasticity of $7.6 \mathrm{cN} /$ tex, tenacity at break of $16.2 \mathrm{cN} / \mathrm{tex}$, and elongation at break of $6 \%$. The addition of carbon nanomaterials did not significantly affect the value of the modulus of elasticity, but it greatly reduced the strength and elasticity of the fibers. The observed negative phenomenon was largely the result of changes in the supramolecular structure. The insignificant increase in the degree of crystallinity along with the significant change in the $d$-spacing and average size of the crystalline area indicated the unfavorable structural reordering of alginic acid from a mechanical properties' point of view. The main changes in the supramolecular structure of alginic acid resulting from the addition of carbon nanomaterials were the increase in $L_{(\mathrm{hkl})}$ corresponding to the (110) crystalline plane of guluronan and the decrease in $L_{(\mathrm{hkl})}$ corresponding to the (200) crystalline plane of mannumoronan. In our opinion, this structural change may indicate a change in the macromolecular order along the fiber axis and explain the visible decline in the mechanical characteristics. Notably, both carbon nanomaterials caused similar effects in the case of alginic acid fibers.

Slightly different changes were observed in the modification of calcium alginate fibers by the addition of carbon nanomaterials. The addition of MWCNTs deteriorated and GO improved the mechanical properties of the studied materials. Along this line, we can interpret the observed phenomena based on the WAXD results. The addition of MWCNTs caused the creation of a highly crystalline structure with fine crystallites (low values of $L_{(\mathrm{hkl})}$ ), resulting in brittle fibers. Quite different results were obtained for calcium alginate modified by the addition of GOs. The implementation of $\mathrm{GO}$ inside the polymer structure caused an increase in the crystallinity and average size of the crystalline area, which is a positive phenomenon causing an increase in the material strength. In addition, calcium alginate containing GO became more stretchable, which may have resulted from the increased $d$-spacing.

It was found that the mechanical properties of alginate fibers strongly depend on the supramolecular structure, which can be modified by the addition of carbon nanomaterials. Importantly, the changes depend on the type of alginate fibers and the type and shape of the nanoadditives. For alginic acid fibers, both nanoadditives caused supramolecular changes and deteriorated the mechanical properties. The presence of GOs in the calcium alginate structure led to positive changes in the mechanical properties.

As noted from the acquired WAXD diffractograms, calcium alginate fibers mostly consist of an amorphous phase, as confirmed by the two dominant components of the amorphous halos at $2 \theta$ values of $22^{\circ}$ and $42^{\circ}$. In turn, the spectra recorded for the alginic acid fibers correspond to a highly ordered rhombic structure of "pure" alginic acid crystallites. The structural modification and increased ordering are attributable to the removed calcium ions in the fibers, as shown by the FTIR results. Generally, the presence of calcium has an adverse effect on the degree of crystallinity of alginate fibers. The resulting "egg-box" structure between guluronate units decreases the conformational freedom of the other macromolecules, including the mannuronate units, and hinders their ordering. ${ }^{32}$ The crystallites formed in the presence of calcium are characterized by a pseudohexagonal arrangement, which is less perfect than the typical rhombic arrangement of alginic acid.

\section{CONCLUSIONS}

The performed experiments and analyses show the scale of the influence of popular carbon nanoadditives on the molecular and supramolecular structures of alginate fibers obtained by the wet-spinning process. This investigation give insights into the analysis of the influence of the shape and chemical composition of nanofillers on the physical properties of the final materials.

The addition of chemically functionalized carbon nanomaterials in the polymer matrix causes an insignificant change in the molecular structure of the material. According to the FTIR spectra, the observed changes likely result from the functional groups of the carbon materials in the polymer matrix, followed by molecular changes of calcium alginate or alginic acid.

The addition of carbon nanomaterials to alginate materials induces ordering of the supramolecular structure of the materials. With the addition of nanofiller, the calcium alginate materials show greater crystallization than the alginic acid materials, which is probably due to the higher orientation of the structure of the unmodified materials. The performed investigation also indicates the influence of the shape and type of applied nanomaterial on the crystallization and structural ordering of the alginates. The addition of MWCNTs results in materials with higher crystallinity and smaller-sized crystalline areas.

The addition of chemically functionalized carbon nanomaterials in the alginate fibers should affect the strength of the materials which corresponds to changes in the supramolecular structure. In contrast, there was no significant effect of molecular changes in alginates on the mechanical properties of the fibers.

The previous research has shown the possibility of incorporating the nanofillers into the material of alginate PCFs. It is worth noting that the introduction of GO into the polymer matrix, despite the occurrence of the agglomeration of these nanoadditives, results in a slight increase in the specific strength of fibers and increase in elongation at break in the case of calcium alginate fibers. For those fibers containing GO, the specific strength is $20 \mathrm{cN} /$ tex which is the highest from all the prepared samples.

The modification of the fibers with hydrochloric acid was aimed at limiting the amount of $\mathrm{Ca}^{2+}$ ions that may adversely affect the prospective carbonization process. Surprisingly, it resulted in a decrease in the specific strength for fibers containing nanoadditives, by almost $50 \%$. At the same time, the fibers after modification with hydrochloric acid show much lower elongation at break.

When analyzing the supermolecular structure of the obtained fibers, it should be emphasized that the calcium alginate fibers are characterized by a pseudohexagonal structure, whereas 
after acid modification, the fibers exhibit a highly crystalline rhombic structure typical of pure alginic acid fibers which influences the mechanical properties adversely. The general finding is that the nanofillers introduced into the polymer matrix induced the nucleation of crystals, causing a slight increase in the degree of crystallinity of both the pseudohexagonal structure of calcium alginate and the highly crystalline rhombic structure of alginic acid.

The current study brings interesting insights into the structureproperty relationship of prospective carbon precursor fibers based on abundantly available biomass-based polymers. These finding provides a basis for further research in the field functionalization of alginate-based PCFs and could provide a significant input for the carbon industry in general.

\section{ACKNOWLEDGMENT}

This study was financed by the National Science Center in Poland awarded on the basis of the decision number DEC2012/05/B/ST8/00249.

\section{References}

[1] Cook, J. G. (1984). Handbook of textile fibres: man-made fibres, Merrow. McGraw Hill Professional (New York).

[2] Haug, A., Smidsrød, O. (1965). Fractionation of alginates by precipitation with calcium and magnesium ions. Acta Chemica Scandinavica, 19, 1221-1226.

[3] Martinsen, A., Skjåk-Bræk, G., Smidsrød, O., Zanetti, F., Paoletti, S. (1991). Comparison of different methods for determination of molecular weight and molecular weight distribution of alginates. Carbohydrate Polymers, 15, 171193.

[4] Grant, G. T., Morris, E. R., Rees, D. A., Smith, P. J. C., Thom, $D$. (1973). Biological interactions between polysaccharides and divalent cations: the egg-box model. FEBS Letters, 32, 195-198.

[5] Li, D., Lu, D., Liu, L., Xia, Y., She, X., et al. (2015). Eggbox structure in cobalt alginate: a new approach to multifunctional hierarchical mesoporous $\mathrm{N}$-doped carbon nanofibers for efficient catalysis and energy storage. ACS Central Science, 1, 261-269.

[6] Zhang, S., Xu, K., Darabi, M. A., Yuan, Q., Xing, M. (2016). Mussel-inspired alginate gel promoting the osteogenic differentiation of mesenchymal stem cells and antiinfection. Materials Science and Engineering Part C, 69, 496-504.

[7] Caetano, L. A., Almeida, A., Gonçalves, L. M. D. (2016). Effect of experimental parameters on alginate/chitosan microparticles for BCG encapsulation. Marine Drugs, 14(5), 90.

[8] Muzzarelli, R. A. A, El Mehtedi, M., Bottegoni, C., Aquili, A., Gigante, A. (2015). Genipin-crosslinked chitosan gels and scaffolds for tissue engineering and regeneration of cartilage and bone. Marine Drugs, 13(12), 7314-7338.

[9] Straccia, M. C., Gomez d'Ayala, G., Romano, I., Oliva, A., Laurienzo, P. (2015). Alginate hydrogels coated with chitosan for wound dressing. Marine Drugs, 13(5), 28902908.
[10] Dhall, S., Silva, J. P., Liu, Y., Hrynyk, M., Garcia, M., et al. (2015). Release of insulin from PLGA-alginate dressing stimulates regenerative healing of burn wounds in rats. Clinical Science, 129(12), 1115-1129

[11] Shamshina, J. L., Gurau, G., Block, L. E., Hansen, L. K., Dingee, C., et al. (2014). Chitin-calcium alginate composite fibers for wound care dressings spun from ionic liquid solution. Journal of Materials Chemistry B, 2(25), 39243936.

[12] Pabjańczyk-Wlazlo, E., Szparaga, G., Król, P., Skrzetuska, E., Wojtasik, K., et al. (2014). Sodium alginate fibers containing nanosilver. Advances in Polymer Technology, 33(S1), 21450.

[13] Wanawananon, K., Moulton, S. E., Wallace, G. G., Liawruangrath, S. (2016). Fabrication of novel core-shell PLGA and alginate fiber for dual-drug delivery system. Polymers for Advanced Technologies - in press

[14] Wu, H. -L., Hou, X. -X., Branford-White, C., Sun, X. -Z., Tao, L., et al. (2015). Drug-loaded microparticles prepared by the one-step deposition of calcium carbonate/alginate onto cotton fabrics. Journal of Applied Polymer Science, 132(40), 42618.

[15] Sun, J., Tan, H. (2013), Alginate-based biomaterials for regenerative medicine applications. Materials, 6(4), 12851309.

[16] Ni, S., Fan, X., Wang, J., Qi, H., Li, X. (2014). Biodegradable implants efficiently deliver combination of paclitaxel and temozolomide to glioma C6 cancer cells in vitro. Annals of Biomedical Engineering, 42(1), 214-221.

[17] Acarregui, A., Orive, G., Pedraz, J. L., Hernández, R. M. (2013). Therapeutic applications of encapsulated cells. Methods In Molecular Biology, 1051, 349-364.

[18] Lee, K. Y., Jeong, L., Kang, Y. O., Lee, S. J., Park, W. H. (2009). Electrospinning of polysaccharides for regenerative medicine. Advanced Drug Delivery Reviews, 61(12), 10201032.

[19] Bouhadir, K. H., Lee, K. Y., Alsberg, E., Damm, K. L., Anderson, K. W., et al. (2001). Degradation of partially oxidized alginate and its potential application for tissue engineering. Journal of Biotechnology Progress, 17, 945950

[20] Mierisch, C. M., Cohen, S. B., Jordan, L. C., Robertson, P. G., Balian, G., et al. (2002). Transforming growth factor- $\beta$ in calcium alginate beads for the treatment of articular cartilage defects in the rabbit. The Journal of Arthroscopic and Related Surgery, 18, 892-900.

[21] Alsberg, E., Anderson, K. W., Albeiruti, A., Franceschi, R. T., Mooney, D. J. (2001). Cell-interactive alginate hydrogels for bone tissue engineering. Journal of Dental Research, 80, 2025-2029.

[22] Chung, T. W., Yang, J., Akaike, T., Cho, K. Y., Nah, J. W., et al. (2002). Preparation of alginate/galactosylated chitosan scaffold for hepatocyte attachment. Biomaterials, 23, 2827-2834.

[23] Jalali, F., Ardeshiri, M. (2016). Application of carbon nanotubes-ionic liquid hybrid in a sensitive atorvastatin ion-selective electrode. Materials Science and Engineering C, 69, 276-282.

[24] Zhang, D. -Y., Ge, C. -W., Wang, J. -Z., Zhang, T. -F., Wu, Y. -C., et al. (2016). Single-layer graphene-TiO2 nanotubes array heterojunction for ultraviolet photodetector application. Applied Surface Science, 387, 1162-1168. 
[25] Bao, J., Hou, C., Dong, Q., Ma, X., Chen, J., et al. (2016). ELP-OPH/BSA/TiO2 nanofibers/c-MWCNTs based biosensor for sensitive and selective determination of p-nitrophenyl substituted organophosphate pesticides in aqueous system. Biosensors and Bioelectronics, 85, 935942.

[26] Tian, W., Zhang, H., Duan, X., Sun, H., Tade, M. O., et al. (2016). Nitrogen- and sulfur-codoped hierarchically porous carbon for adsorptive and oxidative removal of pharmaceutical contaminants. ACS Applied Materials and Interfaces, 8(11), 7184-7193.

[27] Wu, J., Zhao, H., Chen, R., Pham-Huy, C., Hui, X., et al. (2016). Adsorptive removal of trace sulfonamide antibiotics by water-dispersible magnetic reduced graphene oxideferrite hybrids from wastewater. Journal of Chromatography B, 1029-1030, 106-112.

[28] Rabiej, M. (2003). Application of the genetic algorithms and multi-objective optimisation to the resolution of X-Ray diffraction curves of semicrystalline polymers. Fibres and Textiles in Eastern Europe, 11, 83-87.

[29] Rabiej, M. (2003). Application of multicriterial optimization of crystallinity degree of semicrystalline polymers. Polimery, 48, 288-295.

[30] Brzezińska, M., Szparaga, G. (2015). The effect of sodium alginate concentration on the rheological parameters of spinning solutions. Autex Research Journal, 15(2), 123126
[31] Boguń, M. (2009). Rheological properties of sodium alginate spinning solutions with ceramic nanoadditives. Fibres and Textiles in Eastern Europe, 17(5), 17-22.

[32] Boguń, M., Rabiej, S. (2010). The influence of fiber formation conditions on the structure and properties of nanocomposite alginate fibers containing tricalcium phosphate or montmorillonite. Polymers and Composites, 31, 1321-1331.

[33] Arnott, S., Bian, W., Chandrasekaran, R., Manis, B. (2000). Lessons for today and tomorrow from yesterday - the structure of alginic acid. Fibre Diffraction Review, 36, 4451.

[34] Fabia, J., Ślusarczyk, C., Gawłowski, A. (2005). Supermolecular structure of alginate fibres for medical application studied by means of WAXS and SAXS Methods. Fibres and Textiles in Eastern Europe, 53, 114-117.

[35] Gojny, F. H., Wichmann, M. H. G., Kopke, U., Fiedler, B., Schulte, K. (2004). Carbon nanotube reinforced epoxycomposites: enhanced stiffness and fracture toughness at low nanotube content. Composites Science and Technology, 64(15), 2363-71. 